\title{
Parallel-in-time optical simulation of history states
}

\author{
D. Pabón, ${ }^{1,2}$ L. Rebón,,${ }^{3,4,}$ S. Bordakevich, ${ }^{1}$ N. Gigena, ${ }^{3,4}$ A. Boette, ${ }^{3,4}$ C. Iemmi, ${ }^{1,2}$ R. Rossignoli, ${ }^{3,4,5}$ and S. Ledesma ${ }^{1,2}$ \\ ${ }^{1}$ Departamento de Física, FCEyN, Universidad de Buenos Aires, Pabellón 1, Ciudad Universitaria, Buenos Aires 1428, Argentina \\ ${ }^{2}$ Consejo Nacional de Investigaciones Científicas y Técnicas (CONICET), Argentina \\ ${ }^{3}$ Departamento de Física, FCE, Universidad Nacional de La Plata, C.C. 67, La Plata 1900, Argentina \\ ${ }^{4}$ Instituto de Física de La Plata, UNLP-CONICET, Argentina \\ ${ }^{5}$ Comisión de Investigaciones Científicas de la Provincia de Buenos Aires (CIC), La Plata 1900, Argentina
}

(Received 15 January 2019; published 25 June 2019)

\begin{abstract}
We present an experimental optical implementation of a parallel-in-time discrete model of quantum evolution, based on the entanglement between the quantum system and a finite-dimensional quantum clock. The setup is based on a programmable spatial light modulator which entangles the polarization and transverse spatial degrees of freedom of a single photon. It enables the simulation of a qubit history state containing the whole evolution of the system, capturing its main features in a simple and configurable scheme. We experimentally determine the associated system-time entanglement, which is a measure of distinguishable quantum evolution, and also the time average of observables, which in the present realization can be obtained through one single measurement.
\end{abstract}

DOI: 10.1103/PhysRevA.99.062333

\section{INTRODUCTION}

Physics is a science that attempts to describe the behavior of natural systems, i.e., their evolution through time. In classical mechanics time is treated as an external classical parameter, an assumption that remains in the standard formulation of quantum mechanics since probabilities are only assigned to observable measures made at a certain moment in time. In this sense, time reserves a special status in quantum mechanics.

The Newtonian notion of time, in which it is considered as a parameter essentially different from space coordinates, was modified with the introduction of Lorentz transformations in relativity theory, but for each inertial frame it remains as a global external background parameter. In both cases, furthermore, it is assumed that the time coordinate can be read from an appropriate classical clock. This assumption fails in quantum gravity, where the space-time metric is a dynamical object and must therefore be quantized, implying that a physical clock should be a quantum system itself [1-6]. Indeed, as predicted by the Wheeler-DeWitt equation [7], in quantum gravity "there is no time." Canonical quantization of general relativity preserves the constraint of a static state of the universe, and this lead essentially to the problem of time: the incompatibility between a timeless static description of the universe and the notion of time in the evolution of quantum systems.

In the early 1980s Page and Wootters proposed a mechanism [8] to reconcile this apparent contradiction and since then the incorporation of time in a fully quantum framework has attracted increasing attention [9-19]. According to this timeless approach the universe is in a stationary state, and quantum evolution is explained by the entanglement between an evolving subsystem of the universe and a second quantum

\footnotetext{
*rebon@ fisica.unlp.edu.ar
}

system, chosen as the reference clock. The ensuing history state contains the information about the whole evolution of the subsystem, which can be recovered through appropriate measurements at the clock.

An experimental illustration of these ideas is presented in Ref. [20] using the polarization entangled state of two photons, one of which is used as a two-dimensional clock to gauge the evolution of the second. More recently this realization has been extended to use the position of a photon, as a continuous variable, to describe time [21].

On the other hand, a fully discrete version of the formalism, based on a finite-dimensional quantum clock, is developed in Refs. $[18,19]$. Such a scheme leads to discrete history states, which have the advantage that they can be directly generated through a quantum circuit. Moreover, the associated Schmidt decomposition and the ensuing system-time entanglement can be easily obtained, with the latter representing a measure of distinguishable quantum evolution.

In the present work we introduce a simple optical implementation of such a parallel-in-time discrete model of quantum evolution, in which the quantum clock has a finite configurable dimension $N$. This realization is carried out by using the polarization and the transverse spatial degrees of freedom (DOFs) of the light field to encode the emulated bipartite quantum system. Through the use of a programmable spatial light modulator (SLM) we generate nonseparable states sometimes called classical entangled states. The scheme enables the generation of discrete history states of a qubit, and hence allows us to experimentally determine related quantities which characterize the quantum evolution, such as the associated system-time entanglement. Moreover, it allows us to recover time averages of observables of the system efficiently through one single measurement, instead of a set of $N$ sequential measurements.

The paper is organized as follows: We first provide, in Sec. II, a succinct description of the discrete formalism 
presented in Refs. [18,19]. The experimental implementation and results are described in Sec. III, where the modulation introduced by the SLM is analyzed in detail and expressed as unitary operators in polarization space. Theoretical and experimental results for time averages are determined and compared. The ensuing system-clock entanglement is also analyzed for different trajectories, and the so-called entangling power of the setup is discussed as well. Conclusions and perspectives are finally presented in Sec. IV.

\section{FORMALISM}

We consider a system $S$ and a reference clock system $T$ in a joint pure state $|\Psi\rangle \in \mathcal{H}_{S} \otimes \mathcal{H}_{T}$, with $\mathcal{H}_{T}$ of finite dimension $N$. Any such state can be written as $[18,19]$

$$
|\Psi\rangle=\frac{1}{\sqrt{N}} \sum_{t}\left|\psi_{t}\right\rangle|t\rangle,
$$

where $\{|t\rangle\}_{t=0}^{N-1}$ is an orthonormal basis of $T$ and $\left|\psi_{t}\right\rangle$ are states of $S$ (not necessarily orthogonal) satisfying $\sum_{t}\left\langle\psi_{t} \mid \psi_{t}\right\rangle / N=$ $\langle\Psi \mid \Psi\rangle=1$. The state $|\Psi\rangle$ can describe, for instance, the whole evolution of an initial pure state $\left|\psi_{0}\right\rangle$ of a physical system $S$ at a discrete set of times, in which case $\left|\psi_{t}\right\rangle$ is the normalized state of the system at time $t$. Then, $\left|\psi_{t}\right\rangle$ can be recovered as the conditional state of $S$ after a local measurement at $T$ in the previous basis, with result $t$ : If $\Pi_{t}=\mathbb{1} \otimes|t\rangle\langle t|$, then

$$
\left|\psi_{t}\right\rangle\left\langle\psi_{t}\right|=\frac{\operatorname{Tr}_{T}\left(|\Psi\rangle\langle\Psi| \Pi_{t}\right)}{\left\langle\Psi\left|\Pi_{t}\right| \Psi\right\rangle} .
$$

In shorthand notation, $\left|\psi_{t}\right\rangle=\sqrt{N}\langle t \mid \Psi\rangle$. Moreover, if $|\Psi\rangle$ is enforced to be an eigenstate of the unitary operator [19]

$$
\mathcal{U}=\sum_{t} U_{t, t-1} \otimes|t\rangle\langle t-1|,
$$

where $U_{t, t-1}$ are arbitrary unitary operators satisfying the cyclic condition $U_{N, N-1} \ldots U_{1,0}=\mathbb{1}_{S}$ (and $|t=N\rangle \equiv \mid t=$ $0\rangle$ ), then $\left|\psi_{t}\right\rangle$ follows a discrete unitary evolution [19]: $\left|\psi_{t}\right\rangle=$ $U_{t}\left|\psi_{0}\right\rangle$ if $\mathcal{U}|\Psi\rangle=|\Psi\rangle$, with $U_{t}=U_{t, t-1} \ldots U_{1,0}$ (the eigenvalues of $\mathcal{U}$ are the $N N^{\text {th }}$ roots of unity, and $U_{t} \rightarrow e^{-i 2 \pi k t / N} U_{t}$, $k=1, \ldots, N-1$, for the other eigenvalues). Writing $\mathcal{U}=$ $\exp [-i \mathcal{J}]$, the previous eigenvalue equation corresponds to $\mathcal{J}|\Psi\rangle=0$, which is a generalized discrete version of the Wheeler-DeWitt equation [18]. In the special case of a noninteracting $\mathcal{J}$, such that $\mathcal{J}=H_{S} \otimes \mathbb{1}+\mathbb{1} \otimes P_{T}$, then $U_{t}=$ $\exp \left[-i H_{S} t\right]$, with $H_{S}$ being a Hamiltonian for system $S$ and $P_{T}$ a "momentum" for system $T$, both with eigenvalues $2 \pi k / N$. Moreover, the equation $\mathcal{J}|\Psi\rangle=0$ then implies

$$
-\left\langle t\left|P_{T}\right| \Psi\right\rangle=H_{S}\left|\psi_{t}\right\rangle
$$

which in the continuous limit obtained for large $N$ (and setting $\hbar=1$ ) reduces to the Schrödinger equation $i \partial_{t}\left|\psi_{t}\right\rangle=H_{S}\left|\psi_{t}\right\rangle$ [19].

The entanglement of the history state (1) is a measure of the distinguishable evolution undergone by the system [18]. If all states $\left|\psi_{t}\right\rangle$ are orthogonal, then $|\Psi\rangle$ is maximally entangled, whereas if all $\left|\psi_{t}\right\rangle$ are proportional (i.e., a stationary state), then $|\Psi\rangle$ becomes separable. Its entanglement entropy

$$
\begin{gathered}
E(S, T)=\mathcal{S}\left(\rho_{S}\right)=\mathcal{S}\left(\rho_{T}\right), \\
\mathcal{S}(\rho)=-\operatorname{Tr} \rho \log _{2} \rho,
\end{gathered}
$$

where $\rho_{S, T}=\operatorname{Tr}_{T, S}|\Psi\rangle\left\langle\Psi\left|=\sum_{k} \lambda_{k}\right| k_{S, T}\right\rangle\left\langle k_{S, T}\right|$ are the reduced system and clock states, respectively, then ranges from 0 for stationary states to $\log _{2} N$ when all $\left|\psi_{t}\right\rangle$ are mutually orthogonal. Thus, $2^{E(S, T)}$ is a measure of the number of distinguishable states visited by the system. Of course, when the system dimension $d_{S}$ is smaller than $N$, as will occur in the situation here considered, the maximum number of orthogonal states $\left|\psi_{t}\right\rangle$ is $d_{S}$ and hence $E(S, T) \leqslant \log _{2} d_{S}$. In general $E(S, T) \leqslant \log _{2} M$, where $M \leqslant \operatorname{Min}\left[d_{S}, N\right]$ is the rank of $\rho_{S}$ or $\rho_{T}$ (identical). When $S$ is a qubit, $d_{S}=2$ and then $E(S, T) \leqslant \log _{2} d_{S}=1$.

We may also employ the quadratic entanglement

$$
\begin{aligned}
E^{(2)}(S, T) & =\mathcal{S}_{2}\left(\rho_{S}\right)=\mathcal{S}_{2}\left(\rho_{T}\right) \\
& =\frac{2}{N}\left(N-1-\frac{2}{N} \sum_{t<t^{\prime}}\left|\left\langle\psi_{t} \mid \psi_{t^{\prime}}\right\rangle\right|^{2}\right),
\end{aligned}
$$

where $\mathcal{S}_{2}(\rho)=2 \operatorname{Tr} \rho(\mathbb{1}-\rho)=2\left(1-\operatorname{Tr} \rho^{2}\right)$ is the quadratic entropy [also known as linear entropy, as it corresponds to $-\ln \rho \approx \mathbb{1}-\rho$ in $S(\rho)]$. This entropy can be directly evaluated without knowledge of the eigenvalues and can be accessed experimentally through purity measurements of the reduced state $\rho_{S}$. It is again a measure of the distinguishability between the evolved states. Its minimum value for an evolution between fixed initial and finial states due to a constant Hamiltonian $H_{S}$ is obtained for an evolution within the subspace generated by the initial and final states [19], which proceeds precisely along the geodesic determined by the Fubini-Study metric [22-25].

While Eq. (5) is independent of the order of the states $\left|\psi_{t}\right\rangle$, it is also possible to consider the entanglement entropies $E_{n}(S, T)$ associated with the first $n \leqslant N$ time-steps, determined by the partial history states $\left|\Psi_{n}\right\rangle=\frac{1}{\sqrt{n}} \sum_{t=0}^{n-1}\left|\psi_{t}\right\rangle|t\rangle$. Their variation with $n$ will provide information on the type of evolution. For instance, a periodic evolution will lead to an essentially $n$-independent entanglement $E_{n}(S, T)$ (for a periodic evolution of period $L$, such that $\left|\psi_{t+L}\right\rangle=e^{i \gamma_{t}}\left|\psi_{t}\right\rangle$ for $t=0, \ldots, L-1$, the entanglement over $k L$ times is independent of the number of cycles $k: E_{k l}(S, T)=E_{L}\left(S, T_{L}\right)$ [18]), while a steadily increasing $E_{n}(S, T)$ indicates increasing distinguishability of the visited states.

\section{EXPERIMENTAL IMPLEMENTATION}

To provide an experimental realization of the concepts here discussed, we propose a full-optical architecture to generate the discrete history states of Eq. (1). We use the linear transverse-momentum position of single photons to set the time $|t\rangle$ of the quantum clock system $T$ and its polarization to encode the state $\left|\psi_{t}\right\rangle$ of the quantum system $S$. It should be noted that, by encoding the subsystems in two different DOFs of a single particle [26-30], the resulting nonseparable state is not, strictly speaking, a nonlocal quantum entangled state: although such encoding is often referred to as "entanglement" 


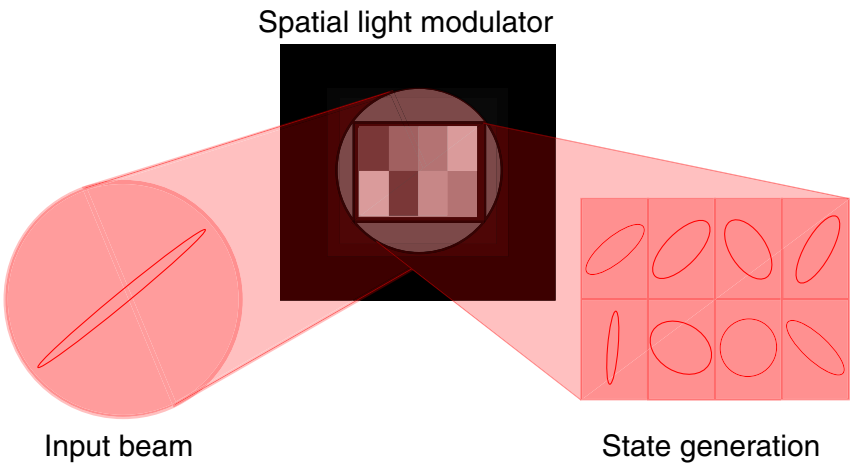

FIG. 1. Sketch of the action of the SLM on the initial state of light. The complex function $\Gamma(\mathbf{x})$ programed on the SLM defines eight spatial regions in the region of interest, and each of them modifies the polarization state according to the particular gray level.

between DOFs, it has a local nature, while "true" quantum entanglement occurs between different particles [31,32].

One of the simplest ways to accomplish this encoding is to use a programmable SLM as a means to create correlations between polarization and spatial DOFs of photons [33-35]. In general, this kind of device allows one to coherently modulate the amplitude, phase, and polarization of the electromagnetic field. It is thus possible to display different regions on the SLM screen and vary, in each of these regions, the polarization of the light field keeping constant its amplitude and phase. It leads to a state generation scheme like that indicated in Fig. 1 where, as an example, eight independent rectangular regions are addressed on the SLM, each one with a different constant function modulation.

\section{A. Generation of discrete history states}

The history state $|\Psi\rangle$ in Eq. (1) can be generated from an initial product state $\left|\psi_{0}\right\rangle|0\rangle$ as

$$
|\Psi\rangle=\mathcal{W}(\mathbb{1} \otimes H)\left|\psi_{0}\right\rangle|0\rangle,
$$

where $H$ is a Hadamard-like gate on the clock system $(H|0\rangle=$ $\left.\frac{1}{\sqrt{N}} \sum_{t=0}^{N-1}|t\rangle\right)$ and $\mathcal{W}=\sum_{t=0}^{N-1} U_{t} \otimes|t\rangle\langle t|$ is the control- $U_{t}$ gate. For $t=0,1, \ldots, N-1$, the $U_{t}$ 's are unitary operators on the system $S$, so that $\left|\psi_{t}\right\rangle=U_{t}\left|\psi_{0}\right\rangle$.

In our experimental implementation, the initial state is a photonic state defined by the product of its polarization state $\left(\left|\psi_{0}\right\rangle\right)$ and its spatial state $(|0\rangle)$ described by the transverse wave-front profile. By using a formalism similar to that of previous works $[35,36]$ where the polarization or the transverse spatial DOFs of photons are manipulated through the use of SLMs, the generation of history states can be explained as follows.

A paraxial and monochromatic single-photon field, assumed here to be in a pure state, is described by

$$
\left|\psi_{0}\right\rangle|0\rangle=\sum_{\mu} \int d \mathbf{x} \alpha_{\mu} f(\mathbf{x})|\mu\rangle|1 \mathbf{x}\rangle,
$$

where $\mu$ runs over two orthogonal polarizations, $\mathbf{x}=(x, y)$ is the transverse position coordinate, and $f(\mathbf{x})$ is the normalized transverse probability amplitude for this state, i.e., $\int d \mathbf{x}|f(\mathbf{x})|^{2}=1$. The SLM introduces a polarizationdependent modulation that can be ideally interpreted as the action of the operator

$$
\Gamma=\sum_{\alpha, \beta} \int d \mathbf{x} \Gamma_{\alpha \beta}(\mathbf{x})|\alpha, 1 \mathbf{x}\rangle\langle\beta, 1 \mathbf{x}|,
$$

so that, after impinging the SLM, the state of the photon field reads

$$
|\Psi\rangle \propto \sum_{\mu, \nu} \int d \mathbf{x} \alpha_{\nu} f(\mathbf{x}) \Gamma_{\mu \nu}(\mathbf{x})|\mu\rangle|1 \mathbf{x}\rangle .
$$

Let us consider the modulation distribution $\Gamma_{\mu \nu}(\mathbf{x})$ defining an array of $N$ rectangular and adjacent spatial regions of width $2 a$ and length $2 b$. On each of these regions we have a constant complex modulation, $C_{\mu \nu}^{(t)}$. Thus,

$$
\begin{aligned}
\Gamma_{\mu \nu}(\mathbf{x}) & =\sum_{t=0}^{N-1} C_{\mu \nu}^{(t)} \operatorname{rect}\left(\frac{x-x_{t}}{2 a}\right) \operatorname{rect}\left(\frac{y-y_{t}}{2 b}\right), \\
C_{\mu \nu}^{(t)} & =c_{\mu \nu}^{(t)} \mathrm{e}^{i \gamma_{\mu \nu}^{(t)}}, \quad c_{\mu \nu}^{(t)} \geqslant 0,
\end{aligned}
$$

where $\operatorname{rect}(u)=1$ if $|u|<\frac{1}{2}, 0$ in the other case, and the centers of these regions are in $\left\{\left(x_{t}, y_{t}\right)\right\}_{t=0}^{N-1}$, with $x_{t}=$ $a, 3 a, 5 a, \ldots$, and $y_{t}=b, 3 b, 5 b, \ldots$. With this prescription we can define the spatial states

$$
|t\rangle=\frac{1}{\sqrt{\mathcal{N}_{t}}} \int d \mathbf{x} \operatorname{rect}\left(\frac{x-x_{t}}{2 a}\right) \operatorname{rect}\left(\frac{y-y_{t}}{2 b}\right)|1 \mathbf{x}\rangle,
$$

which form an orthonormal basis of the discretized spatial Hilbert space of the single photon. Finally, by combining this result with Eq. (12), the transformed state in Eq. (11) can be written in the following way:

$$
|\Psi\rangle \propto \sum_{t=0}^{N-1} \sum_{\mu, v} \alpha_{\nu} C_{\mu \nu}^{(t)}|\mu\rangle|t\rangle .
$$

In our implementation the modulation introduced by the SLM implies a transformation only of the polarization DOF. It means that $\sum_{\mu \nu} \alpha_{\nu} C_{\mu \nu}^{(t)}|\mu\rangle|t\rangle=\left(U_{t} \otimes \mathbb{1}\right)\left|\psi_{0}\right\rangle|t\rangle \equiv\left|\psi_{t}\right\rangle|t\rangle$, with $U_{t}$ being a unitary operator and $\left|\psi_{t}\right\rangle$ the polarization state associated with the $t$-spatial region. Therefore, the SLM transforms the initial photon state as

$$
\begin{aligned}
\left|\psi_{0}\right\rangle|0\rangle \stackrel{\text { SLM }}{\Longrightarrow} \frac{1}{\sqrt{N}} \sum_{t=0}^{N-1} U_{t}\left|\psi_{0}\right\rangle|t\rangle=\mathcal{W}\left(\left|\psi_{0}\right\rangle \sum_{t=0}^{N-1} \frac{1}{\sqrt{N}}|t\rangle\right) \\
=\mathcal{W}(\mathbb{1} \otimes H)\left|\psi_{0}\right\rangle|0\rangle
\end{aligned}
$$

and thus generates the history state as expressed in Eq. (8), where the system $S$ and the clock system $T$ are emulated by the polarization and spatial DOFs, respectively.

\section{B. Setup and measurements}

The experimental setup used for simulating the parallelin-time quantum evolution is sketched in Fig. 2. In the first part, a 660-nm solid state laser beam is expanded, filtered, and collimated in order to illuminate a SLM with a planar wave with approximately uniform amplitude distribution over the region of interest (ROI). This SLM, based on a reflective 


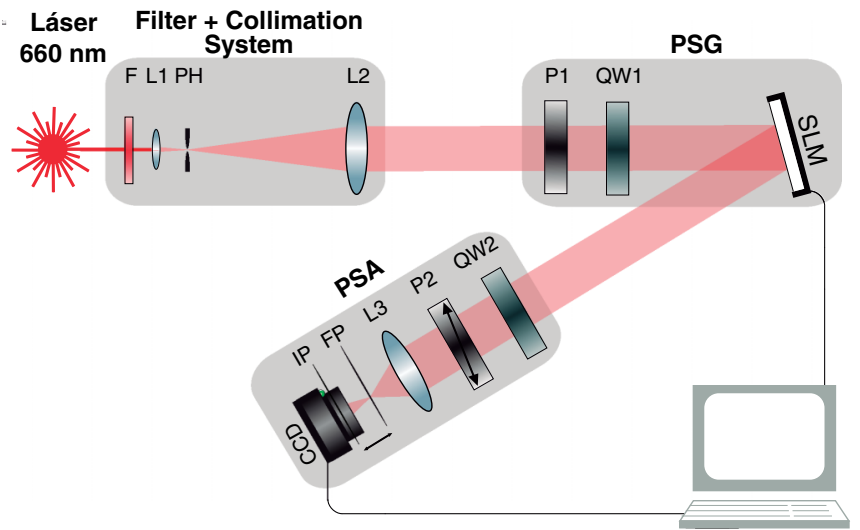

FIG. 2. Experimental setup used for the generation of history states and the subsequent characterization of the evolution of the quantum system $S$.

liquid-crystal-on-silicon (LCoS) microdisplay, with a spatial resolution of $1024 \times 768$ pixels, is used to represent the whole system $|\Psi\rangle$ of Eq. (1). It gives the possibility to dynamically address the optical function on the screen, pixel by pixel. In particular, the SLM used in our experiment, consists of a HoloEye Lc-R 2500 in combination with a polarizer (P1) and a quarter-wave plate (QW1) that provide the adequate incoming state of light to obtain the maximum range of polarization modulation. This is obtained from a MuellerStokes characterization of the LCoS [37,38], followed by an optimization to have a wide range of pure polarization modulation, i.e., without any additional global phase due to an optical path difference, regardless of the gray level that the pixels of the LCoS are set for. Therefore, as each pixel is controlled individually, we can program a particular function $\Gamma(\mathbf{x})$ which characterizes the modulation distribution. Then, the wave front of the electromagnetic field acquires a specific polarization conditioned on the transverse position in the plane of the SLM.

In the second part of the setup, a polarization state analyzer (PSA) is used for the initial characterization of the SLM as a polarization state generation (PSG). For this purpose, after reflection on the SLM, the outgoing beam is focused by the lens L3 onto the detection plane, which is chosen to match the image plane (IP) or the Fourier plane (FP). A quarter-wave plate $(\mathrm{QW} 2)$ and a linear polarizer $(\mathrm{P} 2)$ project the polarization state of the light beam in the different states of the reconstruction basis. Intensity measurements are recorded in the IP or in the FP, depending on the characterization for amplitude or phase modulation, respectively.

In addition, and as a proof-of-principle demonstration, we have inserted neutral-density filters, previous to the PSG stage, to highly attenuate the power of the laser beam at the single-photon regime in such a way that it corresponds to the presence of less than one photon, on average, at any time, in the experiment. This pseudo single-photon source can be used to mimic a single-photon state, and as is usual in optical implementations of quantum simulations or quantumstate estimation [39-41], it is enough to test the feasibility of the proposed method for simulating the main features of a parallel-in-time quantum evolution. Besides, instead of

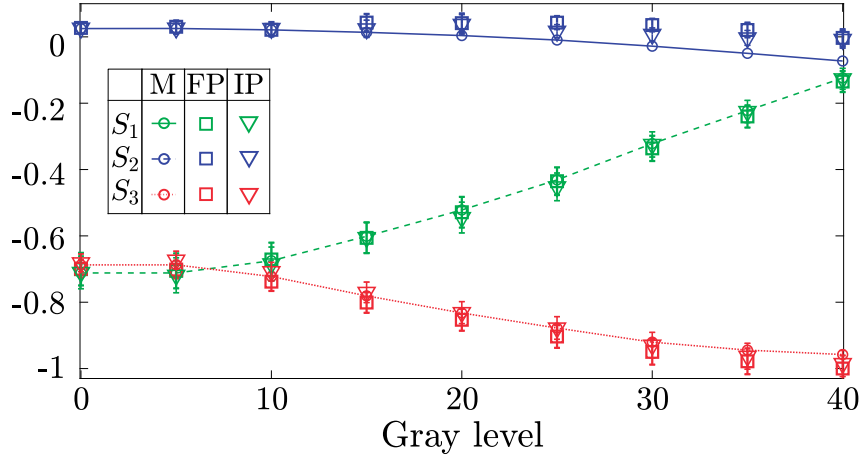

FIG. 3. Normalized Stokes parameters of the polarization state reflected by the SLM as a function of the gray level. The incoming polarization state is given by $\left(S_{0}, S_{1}, S_{2}, S_{3}\right)=$ $(1.000,0.040,0.951,-0.026)$. The graphic shows the experimental values obtained by a measurement in the IP $(\nabla)$ or in the FP $(\square)$, in comparison with those predicted by the Mueller matrix $(\bigcirc)$.

a CCD camera, we used a highly sensitive camera based on complementary metal-oxide semiconductor (CMOS) technology (Andor Zyla 4.2 sCMOS) to carry out the intensity measurements in this regime.

In Fig. 3 we plot the Stokes parameters of the state prepared by the SLM when a single gray level, between 0 and 40, is addressed on the whole screen. In any case the polarization of the input state is $\left(S_{0}, S_{1}, S_{2}, S_{3}\right)=$ $(1.000,0.040,0.951,-0.026)$. Figure 3 shows the parameter values obtained as a measurement in the IP $(\nabla)$ or in the FP ( $\square$ ), in comparison with those predicted by the Mueller matrix $(\bigcirc)$. For these range of gray levels, all the values are in good agreement, which indicates a good performance of the whole setup for the modulation of the polarization state and subsequent characterization of such states. We should mention that, while it is possible to set gray levels up to 255 , for those above 40 the depolarization due to temporal phase fluctuations of the employed SLM becomes important. In fact, devices based on LCoS technology may lead to a flicker in the optical beam because of the digital addressing scheme (pulse width modulation) which introduces, among other undesirable effects, those phase fluctuations $[42,43]$ that affect the quality of the state that it is intended to encode.

Once the modulation of the SLM was fully characterized, the same PSG-PSA system was used to experimentally perform the system-time history state $|\Psi\rangle$ and for the subsequent characterization of the discrete unitary evolution of the system state $\left|\psi_{t}\right\rangle=U_{t}\left|\psi_{0}\right\rangle(t=0, \ldots, N-1)$. For gray levels between 0 and 40 , different history states $|\Psi\rangle$ were generated with two, four, and eight time steps. These history states are displayed in Table I. According to our experimental implementation, each state $\left|\psi_{t}\right\rangle$ visited by the system is specified in terms of the mean values $\left\langle\sigma_{\mu}\right\rangle$ of the Pauli operators $\sigma_{\mu}$, which are just the measured Stokes parameters: $S_{1}=\left\langle\sigma_{z}\right\rangle$, $S_{2}=\left\langle\sigma_{x}\right\rangle$, and $S_{3}=\left\langle\sigma_{y}\right\rangle$ (see Sec. III D). Trajectories 1-4 employ gray levels 0 and 30; trajectories 5-6 employ gray levels $0,15,25$, and 35 ; and trajectory 7 uses gray levels 0,10 , $15,20,25,30,35$, and 40 . These trajectories were chosen in order to compare, for example, "equivalent" (e.g., trajectories 2 and 3 or trajectories 5 and 6 , which differ just in the order 
TABLE I. Generated history states corresponding to two time-steps (trajectory 1), four time-steps (trajectories 2, 3, 5, and 6), and eight time-steps (trajectories 4 and 7). The discrete evolution of the system is seen as a trajectory on the Bloch sphere. The initial state of the system $\left|\psi_{0}\right\rangle$ is described by the Stokes vector $\left(S_{0}, S_{1}, S_{2}, S_{3}\right)=(1.000,0.040,0.951,-0.026)$. In parentheses, we show the gray level used to experimentally implement each trajectory.

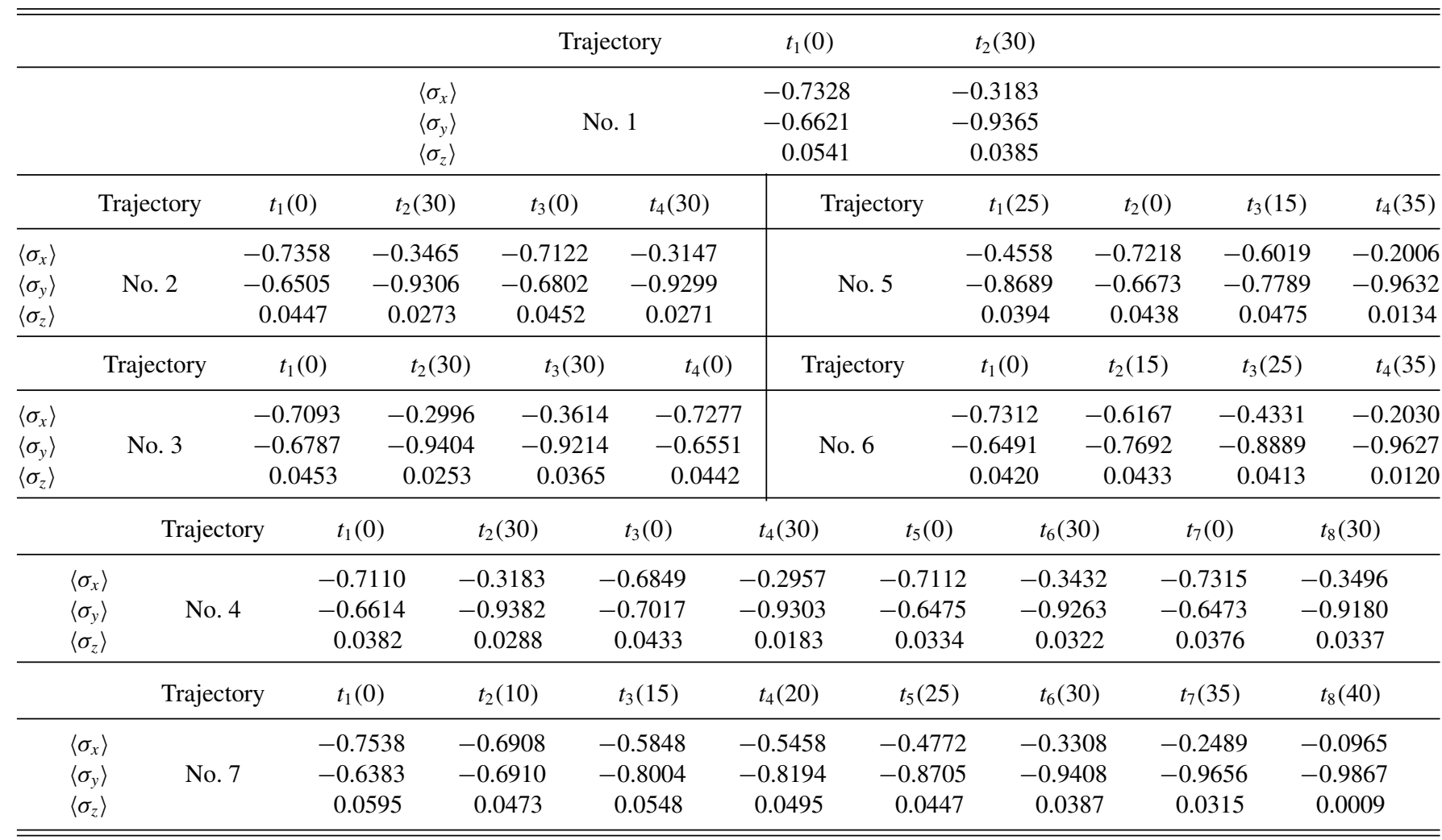

of gray levels) and "nonequivalent" (e.g., trajectories 4 and 7) sets of gray levels, or to compare between essentially periodic (trajectories 2 and 4) and nonperiodic (trajectories 1 and 3) evolutions.

\section{System evolution and mean values}

In the previous subsection we have described how our setup generates history states within a parallel-in-time discrete model of quantum evolution. This implementation allows us to compute the time averages of system observables throughout the system's evolution in two different ways:

(i) from the set of measurements which are performed, sequentially, on the system $S$,

(ii) from a single measurement that involves information of the whole evolution of the system $S$.

In fact, let us consider an operator $A=O \otimes \mathbb{1}$, with $\mathbb{1}$ being the identity operator on the clock system and $O$ an observable of the system $S$. Then, its expectation value in the full history state $|\Psi\rangle$ is given by

$$
\begin{aligned}
\langle A\rangle_{\Psi} & \equiv\langle\Psi|O \otimes \mathbb{1}| \Psi\rangle \\
& =\frac{1}{N} \sum_{t=0}^{N-1}\left\langle\psi_{t}|O| \psi_{t}\right\rangle,
\end{aligned}
$$

which represents the time average $\overline{\langle O\rangle}=\frac{1}{N} \operatorname{Tr}_{S}\left(\sum_{t=0}^{N-1}\right.$ $\left.O\left|\psi_{t}\right\rangle\left\langle\psi_{t}\right|\right)$

In our experimental scheme, we can identify the observable $O$ with one of the Pauli operators $\sigma_{\mu}$. In order to test these two approaches we perform a proper polarization measurement to compute the time average $\overline{\left\langle\sigma_{\mu}\right\rangle}$ for different evolutions of the system $S$. For this purpose the PSA is used to project the polarization state of the incoming beam and record the intensity of the nonextinguished beam. On one hand, if an intensity measurement is performed in the IP, the mean values of the Pauli operators $\sigma_{\mu}$ will vary from one of the spatial regions defined in Eq. (12) to the other, depending on the modulation $C_{\mu \nu}^{(t)}$ assigned to each of these regions. If the polarization state associated with the region $t$ is $\left|\psi_{t}\right\rangle, \sigma_{\mu}$ will have the mean value $\left\langle\psi_{t}\left|\sigma_{\mu}\right| \psi_{t}\right\rangle$ for this region, and the average of the full ROI is then computed as $\frac{1}{N} \sum_{t=0}^{N-1}\left\langle\psi_{t}\left|\sigma_{\mu}\right| \psi_{t}\right\rangle$. On the other hand, if an intensity measurement is performed in the FP, each of the spatial regions addressed on the SLM contribute to build the interference pattern. However, it is not possible to relate a spatial region in the IP to a particular region in the FP. The mean values are then given by $\left\langle\Psi\left|\sigma_{\mu} \otimes \mathbb{1}\right| \Psi\right\rangle$, which implies a global measure in the FP. These two quantities are of course the same, since in the absence of optical losses, the total intensity of the nonextinguished beam is involved in their calculation, as expressed in Eq. (16).

Therefore, if we think of $|\Psi\rangle$ as a history state, our scheme provides an efficient method for the evaluation of the timeaveraged polarization of the system throughout its trajectory. In fact, results shown in Fig. 4 exhibit an excellent agreement between both experimental measurements and between these and the theoretical values. In this plot we can see the time averages $\overline{\left\langle\sigma_{\mu}\right\rangle}$ of the history states described in Table I, which correspond to different evolutions of the same initial state 


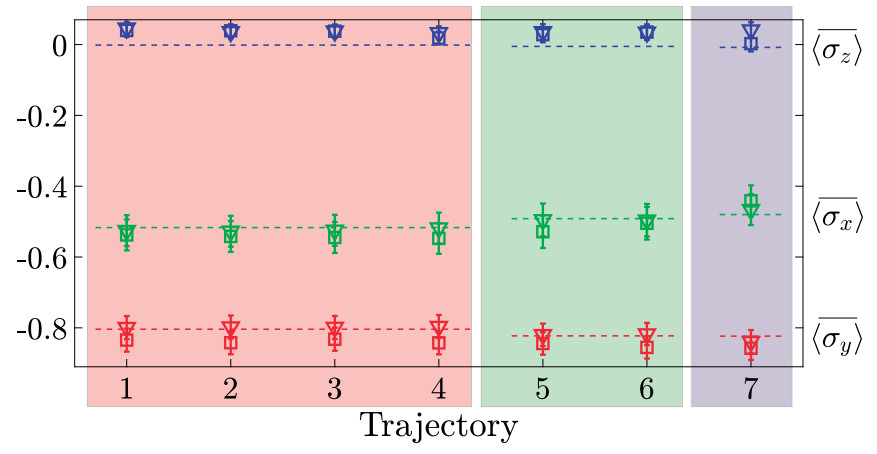

FIG. 4. Time averages of the polarization observables, $\overline{\left\langle\sigma_{\mu}\right\rangle}$, for different evolutions of the initial system state $\left|\psi_{0}\right\rangle$. The graphic shows the predicted values (--) in comparison with the experimental values, obtained by averaging over all spatial regions ( $\square$ ), and by means of one single measurement $(\nabla)$. Each sector, identified by a single color, indicates different evolutions through the same states $\left|\psi_{t}\right\rangle$.

$\left|\psi_{0}\right\rangle$. As expected, these time averages have all the same values for trajectories 1-4 and for trajectories 5-6.

In Sec. III A we stated that the modulation introduced by the SLM can be described by a unitary transformation in polarization space. Experimentally, the modulation associated with a given gray level on the screen is described by a $4 \times 4$ Mueller matrix $M$ [38]. The Mueller matrix acts as a linear transformation on the polarization state of the light field represented by the Stokes vector $S$, defined as

$$
\boldsymbol{S}=\left(\begin{array}{l}
S_{0} \\
S_{1} \\
S_{2} \\
S_{3}
\end{array}\right)=\left(\begin{array}{c}
P_{00}+P_{\frac{\pi}{2} 0} \\
P_{00}-P_{\frac{\pi}{2} 0} \\
P_{\frac{\pi}{4} 0}+P_{-\frac{\pi}{4} 0} \\
P_{\frac{\pi}{4} \frac{\pi}{2}}+P_{-\frac{\pi}{4} \frac{\pi}{2}}
\end{array}\right),
$$

where the vector coefficients $P_{\theta \phi}$ are the results of six polarization measurements: horizontal and vertical linear polarization $\left(P_{00}, P_{\frac{\pi}{2} 0}\right),+45$ and -45 linear polarization $\left(P_{\frac{\pi}{4} 0}, P_{-\frac{\pi}{4} 0}\right)$, and right and left circular polarization $\left(P_{\frac{\pi}{4}} \frac{\pi}{2}, P_{-\frac{\pi}{4} \frac{\pi}{2}}\right)$. Within the quantum formalism, such measurements correspond to projections onto the polarization states $\left|P_{\theta \phi}\right\rangle=\cos (\theta)|H\rangle+$ $e^{i \phi} \sin (\theta)|V\rangle$, so that we have $S_{1,2,3}=\left\langle\sigma_{z, x, y}\right\rangle$, provided $P_{00}+$ $P_{\frac{\pi}{2} 0}=1$ and $\sigma_{\mu}$ 's are the Pauli operators defined with respect to the basis $\{|H\rangle,|V\rangle\}$. The polarization state of a single photon is therefore given by

$$
\rho=\frac{1}{2}(I+\boldsymbol{r} \cdot \boldsymbol{\sigma})
$$

with $\boldsymbol{r}=\frac{1}{S_{0}}\left(S_{1}, S_{2}, S_{3}\right)$, and a unitary transformation in polarization space corresponds then to a rotation of the Bloch vector $\boldsymbol{r}$, which will be associated with a Mueller matrix of the form

$$
M_{R}=\left(\begin{array}{cc}
1 & \mathbf{0} \\
\mathbf{0} & m_{R}
\end{array}\right),
$$

where $m_{R}$ denotes an arbitrary $3 \times 3$ rotation matrix. A Mueller matrix such as that describes the effect of an ideal retarder. However, the SLM used in our implementation introduces not only retardance but also diattenuation. Therefore the Mueller matrix associated with a given gray level will not have the form (19) that maps to a unitary transformation
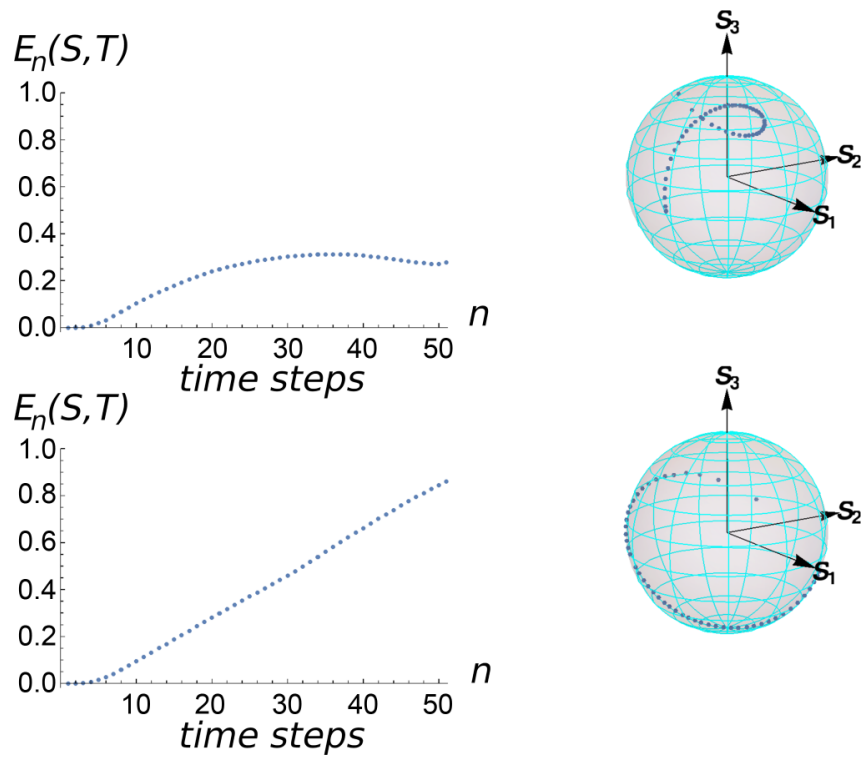

FIG. 5. System-time entanglement entropy $E_{n}(S, T)$ vs number of steps (left panels) and trajectories in the polarization Bloch sphere (right panels) for two different initial states. The same set of 52 unitary evolution operators extracted from the experimental characterization was employed in both cases.

in polarization space. It is possible, nonetheless, to extract from a general Mueller matrix a pure retardance matrix that accounts for the effective phase transformation introduced by the optical system, by means of the Lu-Chipman decomposition [44]. In this way, from the Mueller matrices obtained from the experimental characterization of the SLM we extracted a set of unitary matrices that describe the transformations performed on the polarization of the photon field, for 52 gray levels between 0 and 255, in steps of 5 . The set of unitary matrices described above allows us to simulate history states beyond those that we have actually implemented. The right panels in Fig. 5 show examples of such simulated evolutions of the photon polarization as trajectories on the Bloch sphere.

In Fig. 5, we also depict in the left panels the entanglement entropies $E_{n}(S, T)$ associated with the trajectories determined by the 52 unitaries derived from the $N=52$ experimentally determined Mueller matrices, for two different initial states, as a function of the number $n$ of steps. For $n=N, E_{n}(S, T)$ becomes the system-time entanglement entropy $E(S, T)$ of the full trajectory. In the top and bottom panels time-ordering corresponds to increasing gray levels. In the top panel the trajectory exhibits a loop starting at step $n \approx 35$, implying a decreasing distinguishability between evolved states in this sector, which is reflected in a decrease of $E_{n}(S, T)$ for $n \gtrsim 35$. In contrast, in the bottom panel $E_{n}(S, T)$ increases linearly with $n$ as the trajectory has no loops and does not cross itself.

Figure 6 depicts the entropies $E_{n}(S, T)$ for the two experimental eight-step trajectories of Table I. That on the left stays approximately constant after the third step, since it is determined by a configuration with just two gray levels and the trajectory essentially oscillates between two nonorthogonal states, $\left|\psi_{0}\right\rangle$ and $\left|\psi_{1}\right\rangle$. In this case $\left|\Psi_{n}\right\rangle=$ $\frac{1}{\sqrt{n}}\left[\left|\psi_{0}\right\rangle\left(\sum_{t \text { even }}^{n-1}|t\rangle\right)+\left|\psi_{1}\right\rangle\left(\sum_{t \text { odd }}^{n-1}|t\rangle\right)\right]$ and the exact theoret- 

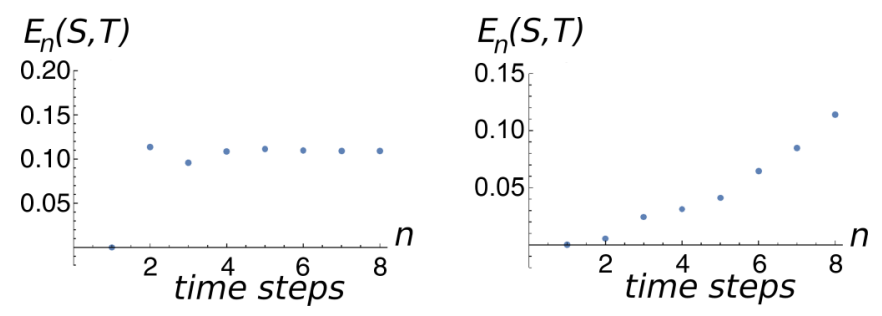

FIG. 6. System-time entanglement entropy $E_{n}(S, T)$ vs number of steps, for the experimentally generated history states corresponding to the two eight-time-step trajectories of Table I.

ical value of $E_{n}(S, T)$ is given by

$$
E_{n}(S, T)=-\sum_{\nu= \pm} p_{n, v} \log _{2} p_{n, v},
$$

where the probabilities $p_{n, \pm}$ are $n$ independent for $n$ even while for $n$ odd they rapidly approach the same even values as $n$ increases:

$$
p_{n, \pm}=\left\{\begin{array}{lc}
\frac{1}{2}\left[1 \pm\left|\left\langle\psi_{0} \mid \psi_{1}\right\rangle\right|\right], & n \text { even } \\
\frac{1}{2}\left[1 \pm \sqrt{\left|\left\langle\psi_{0} \mid \psi_{1}\right\rangle\right|^{2}\left(1-\frac{1}{n^{2}}\right)+\frac{1}{n^{2}}}\right], & n \text { odd }
\end{array}\right.
$$

The observed value $E_{n}(S, T) \approx 0.11$ (for $n$ even or $n \gtrsim 5$ if odd) is then in agreement with the overlap $\left|\left\langle\psi_{0} \mid \psi_{1}\right\rangle\right| \approx 0.97$ between both states. This almost periodic trajectory is compatible with an approximately constant effective Hamiltonian $H=\frac{\pi}{2} \boldsymbol{n} \cdot \boldsymbol{\sigma}$, where $\boldsymbol{n}$ is a vector in the plane spanned by the Bloch vectors of $\left|\psi_{0}\right\rangle$ and $\left|\psi_{1}\right\rangle$, halfway between both states, such that $e^{-i H}$ is a rotation of angle $\pi$ around this axis and $e^{-i H}\left|\psi_{0}\right\rangle=\left|\psi_{1}\right\rangle$ and $e^{-i H}\left|\psi_{1}\right\rangle=\left|\psi_{0}\right\rangle$.

On the other hand, in the right panel of Fig. $6, E_{n}(S, T)$ increases almost linearly for $t \gtrsim 5$, reflecting a trajectory where the distinguishability between the evolved state $\left|\psi_{t}\right\rangle$ and the initial state increases monotonically. In this case the evolved states lie approximately within a plane and the trajectory is approximately compatible with the Hamiltonian $H=\alpha_{t} \boldsymbol{n} \cdot \boldsymbol{\sigma}$, with $\boldsymbol{n}$ orthogonal to this plane and varying strength $\alpha_{t}$ (or equivalently, constant $\alpha_{t}$ and varying time intervals).

We mention that the behavior of the quadratic entropy $E_{n}^{(2)}(S, T)$ is completely similar to that of $E_{n}(S, T)$, since the polarization-reduced state $\rho_{S}$ is a qubit state. For a qubit, $S_{2}\left(\rho_{S}\right)$ is just an increasing (and concave) function of the von Neumann entropy $S\left(\rho_{S}\right)$.

\section{Evolution operators and entangling power}

For any of these simulated history states we can now reconsider the generating operator $\mathcal{W}=\sum_{t} U_{t} \otimes|t\rangle\langle t|$, which can be here expressed as

$$
\mathcal{W}=\sum_{t}\left(\frac{1}{2} \sum_{\mu}^{\prime} r_{\mu}(t) \sigma_{\mu}\right) \otimes|t\rangle\langle t|=\sum_{\mu}^{\prime} \lambda_{\mu} \tilde{\sigma}_{\mu} \otimes O_{\mu} .
$$

Here we have first expanded the unitary operators in polarization space in the Pauli operators plus $\sigma_{0}=\mathbb{1}$, with $r_{\mu}(t)=$ $\operatorname{Tr} U_{t} \sigma_{\mu}$ (and $\sum_{\mu}^{\prime}=\sum_{\mu=0}^{3}$ ), and then written the ensuing Schmidt decomposition [19], where $\tilde{\sigma}_{\mu}$ and $O_{\mu}$ are orthogonal operators in polarization and spatial spaces $\left[\operatorname{Tr}\left(\tilde{\sigma}_{\mu}^{\dagger} \tilde{\sigma}_{\nu}\right)=\right.$ $\left.2 \delta_{\mu \nu}, \operatorname{Tr}\left(O_{\mu}^{\dagger} O_{\nu}\right)=N \delta_{\mu \nu}\right]$. The real non-negative numbers $\lambda_{\mu}$ are the Schmidt coefficients, which are the singular values of the $4 \times N$ matrix $C_{\mu, t}=r_{\mu}(t) / \sqrt{N}$ and satisfy $\sum_{\mu} \lambda_{\mu}^{2}=1$.

Its quadratic operator entanglement [19], $E^{(2)}(\mathcal{W})=$ $2\left(1-\sum_{\mu} \lambda_{\mu}^{4}\right)$, which depends on the unitary evolution operators $U_{t}$ but not on the initial state, is proportional to the entangling power of $\mathcal{W}$ [19], which is the average quadratic entanglement it generates when applied to initial product states $\left|\psi_{0}\right\rangle H^{\otimes n}|0\rangle\left(N=2^{n}\right)$ :

$$
\left\langle E^{(2)}(S, T)\right\rangle=\frac{d_{S}}{d_{S}+1} E^{(2)}(\mathcal{W}),
$$

where $d_{S}$ is the dimension of the system $\left(d_{S}=2\right.$ in the present case), and

$$
\left\langle E^{(2)}(S, T)\right\rangle=\int_{\mathcal{H}} 2\left(1-\operatorname{Tr} \rho_{S}^{2}\right) d \psi_{0}
$$

is the average over all $\left|\psi_{0}\right\rangle$ of the quadratic entanglement entropy $E^{(2)}(S, T)$ of the associated history state, with the integral running over the whole set of initial states $\left|\psi_{0}\right\rangle$ with the Haar measure $d \psi_{0}$ [19]. We have verified this relation by considering the full set of 52 available polarization unitaries extracted from the experimental characterization, which provided a value of $E^{(2)}(\mathcal{W})=0.71$. A simulation with 1000 random initial states satisfied the previous relation with an error of less than 0.01 .

\section{CONCLUSIONS}

We have presented a simple optical implementation for realizing discrete history states. The approach is based on the entanglement between the polarization and spatial DOFs generated by the SLM and can be used to generate history states with a controllable number of time steps for a qubit system. It enables an efficient determination of time averages through a single measurement. The experimental results obtained with the previous scheme show, in fact, an excellent agreement between both the direct method and the sequential method, and also with the theoretical results. The associated "system-clock" entanglement, which is a measure of the distinguishability of the evolved polarization states, was also determined and shown to characterize the basic features of the discrete trajectories obtained for different initial states. The entangling power of the setup, which determines the average quadratic entanglement that it generates when applied to random initial states, was also analyzed. Variations of the present scheme based on two entangled photons could provide a realization of discrete history states of higher-dimensional systems and are currently under development.

\section{ACKNOWLEDGMENTS}

We express our gratitude to Prof. C. T. Schmiegelow for providing us with the sCMOS camera. This work was supported by the Agencia Nacional de Promoción Científica y Tecnológica ANPCyT (Grant No. PICT 2014-2432) and the Universidad de Buenos Aires (Grant No. UBACyT 20020170100040B). R.R. acknowledges support from the CIC of Argentina. 
[1] C. Rovelli, Quantum Gravity, Cambridge Monographs on Mathematical Physics (Cambridge University, Cambridge, England, 2004).

[2] K. V. Kuchař, Int. J. Mod. Phys. D 20, 3 (2011).

[3] C. Isham, in Integrable Systems, Quantum Groups and Quantum Field Theory, edited by L. A. Ibort and M. A. Rodríguez (Kluwer, Dordrecht, 1993), p. 157.

[4] J. Tambornino, SIGMA 8, 017 (2012).

[5] M. Bojowald, P. A. Höhn, and A. Tsobanjan, Phys. Rev. D 83, 125023 (2011).

[6] P. A. Höhn, E. Kubalova, and A. Tsobanjan, Phys. Rev. D 86, 065014 (2012).

[7] B. S. DeWitt, Phys. Rev. 160, 1113 (1967).

[8] D. N. Page and W. K. Wootters, Phys. Rev. D 27, 2885 (1983).

[9] R. Gambini, R. A. Porto, J. Pullin, and S. Torterolo, Phys. Rev. D 79, 041501(R) (2009).

[10] V. Giovannetti, S. Lloyd, and L. Maccone, Phys. Rev. D 92, 045033 (2015).

[11] S. Massar, P. Spindel, A. F. Varón, and C. Wunderlich, Phys. Rev. A 92, 030102(R) (2015).

[12] J. McClean, J. Parkhill, and A. Aspuru-Guzik, Proc. Natl. Acad. Sci. USA 110, E3901 (2013).

[13] J. R. McClean and A. Aspuru-Guzik, Phys. Rev. A 91, 012311 (2015).

[14] P. Erker, M. T. Mitchison, R. Silva, M. P. Woods, N. Brunner, and M. Huber, Phys. Rev. X 7, 031022 (2017).

[15] E. O. Dias and F. Parisio, Phys. Rev. A 95, 032133 (2017).

[16] A. Nikolova, G. K. Brennen, T. J. Osborne, G. J. Milburn, and T. M. Stace, Phys. Rev. A 97, 030101(R) (2018).

[17] P. Coles, V. Katariya, S. Lloyd, I. Marvian, and M. Wilde, Phys. Rev. Lett. 122, 100401 (2019).

[18] A. Boette, R. Rossignoli, N. Gigena, and M. Cerezo, Phys. Rev. A 93, 062127 (2016).

[19] A. Boette and R. Rossignoli, Phys. Rev. A 98, 032108 (2018).

[20] E. Moreva, G. Brida, M. Gramegna, V. Giovannetti, L. Maccone, and M. Genovese, Phys. Rev. A 89, 052122 (2014).

[21] E. Moreva, M. Gramegna, G. Brida, L. Maccone, and M. Genovese, Phys. Rev. D 96, 102005 (2017).

[22] J. Anandan and Y. Aharonov, Phys. Rev. Lett. 65, 1697 (1990).

[23] H. P. Laba and V. M. Tkachuk, Condens. Matter Phys. 20, 13003 (2017).
[24] L. Mandelstam and I. Tamm, J. Phys. USSR 9, 249 (1945).

[25] K. Battacharyya, J. Phys. A 16, 2993 (1983).

[26] N. J. Cerf, C. Adami, and P. G. Kwiat, Phys. Rev. A 57, R1477(R) (1998).

[27] M. Fiorentino and F. N. C. Wong, Phys. Rev. Lett. 93, 070502 (2004).

[28] B. Ndagano, B. Perez-Garcia, F. S. Roux, M. McLaren, C. Rosales-Guzman, Y. Zhang, O. Mouane, R. I. HernandezAranda, T. Konrad, and A. Forbes, Nat. Phys. 13, 397 (2017).

[29] K. H. Kagalwala, G. Di Giuseppe, A. F. Abouraddy, and B. E. Saleh, Nat. Commun. 8, 739 (2017).

[30] P. Imany, J. A. Jaramillo-Villegas, J. M. Lukens, O. D. Odele, D. E. Leaird, M. Qi, and A. M. Weiner, arXiv:1805.04410.

[31] E. Karimi and R. W. Boyd, Science 350, 1172 (2015).

[32] A. Aiello, F. Töppel, C. Marquardt, E. Giacobino, and G. Leuchs, New J. Phys. 17, 043024 (2015).

[33] E. Nagali, L. Sansoni, L. Marrucci, E. Santamato, and F. Sciarrino, Phys. Rev. A 81, 052317 (2010).

[34] R. Fickler, R. Lapkiewicz, S. Ramelow, and A. Zeilinger, Phys. Rev. A 89, 060301(R) (2014).

[35] G. B. Lemos, J. O. De Almeida, S. P. Walborn, P. H. Souto Ribeiro, and M. Hor-Meyll, Phys. Rev. A 89, 042119 (2014).

[36] M. A. Solís-Prosser, A. Arias, J. J. M. Varga, L. Rebón, S. Ledesma, C. Iemmi, and L. Neves, Opt. Lett. 38, 4762 (2013).

[37] A. Márquez, C. Iemmi, I. Moreno, J. A. Davis, J. Campos, and M. J. Yzuel, Opt. Eng. 40, 2558 (2001).

[38] A. Márquez, I. Moreno, C. Iemmi, A. Lizana, J. Campos, and M. J. Yzuel, Opt. Express 16, 1669 (2008).

[39] M. Malik, M. Mirhosseini, M. P. J. Lavery, J. Leach, M. J. Padgett, and R. W. Boyd, Nat. Commun. 5, 3115 (2014).

[40] Q. Pears Stefano, L. Rebón, S. Ledesma, and C. Iemmi, Phys. Rev. A 96, 062328 (2017).

[41] D. Martínez, A. Tavakoli, M. Casanova, G. Cañas, B. Marques, and G. Lima, Phys. Rev. Lett. 121, 150504 (2018).

[42] A. Lizana, I. Moreno, A. Márquez, C. Iemmi, E. Fernández, J. Campos, and M. J. Yzuel, Opt. Express 16, 16711 (2008).

[43] A. Lizana, A. Márquez, L. Lobato, Y. Rodange, I. Moreno, C. Iemmi, and J. Campos, Opt. Express 18, 10581 (2010).

[44] S.-Y. Lu and R. A. Chipman, J. Opt. Soc. Am. A 13, 1106 (1996). 